\title{
32nd ANNUAL SASKATCHEWAN CHRISTMAS MAMMAL COUNT-2004.
}

\section{ALAN R. SMITH, 115 Perimeter Road, Saskatoon SK S7N0X4.}

Mammal counts (Table 1) were received from 95 of the 99 localities submitting Christmas Bird Counts (CBC). The 33 species of mammals seen or heard on count day was second only to last year's 35 species and tied totals for 2002, 1995 and 1991.

The most abundant mammal was the White-tailed Deer, closely followed by the Mule Deer. The American Red Squirrel and Pronghorn placed third and fourth. The most frequently seen mammals involved the White-tailed Deer on $71 \%$ of all counts, the Coyote on 59\%, the American Red Squirrel on $53 \%$, and the Mule Deer on only $38 \%$.

No new species were recorded, but the Least Chipmunk was recorded for only the third time and for the second year in a row. One was seen at Fort Qu'Appelle. The previous records include one seen at Archerwill and tracks noted at Saskatoon in 2003.

Another good find for the Fort Qu'Appelle count was a Short-tailed Shrew. A Fisher on the Spinney Hill count was south of its normal boreal forest range. Although identified only by tracks, a Woodland Caribou at Candle Lake was of interest as was a den considered to be that of a Swift Fox in Grasslands National Park.

For information on participants, weather, coverage and location of Christmas Mammal Counts see the CBC summary in this issue.

The number of mammals actually seen or heard on count day is treated separately from those recorded by other means, or those recorded during count period (14 December to 5 January, as with the CBC) but not on count day. Numbers of individuals seen or heard are given in the table and are tallied in the first line of totals at the bottom of Table 1. The number of species they represent is given in the second line.

For animals detected by tracks, by other means, or that are seen or heard in the count period but not on count day, we tabulate only the species additional to those actually seen or heard and no number of individuals is given. Species detected only by tracks are indicated by ' $t$ ' in the table; those detected only by other means (dead animals or clearly identifiable chewing, digging, dens or lodges) by 'o.' Species detected by any means during the count period, but not on count day are indicated by ' $c$ ' in the table. These additional species are tallied in lines 3,4 and 5 at the bottom of the table. If a mammal is reported as member of a species group (i.e. mouse species, deer species), it is counted as a species only if no other species in this group has been definitely recorded.

The columns at the end of the table give totals for each species. The first shows the total number of individuals seen or heard on count day. The second gives the number of counts on which the species was seen or heard on count day. Columns 3 and 4 give the number of counts on which the species was recorded only by tracks or other signs. Column 5 shows the number of times the species was recorded during the count period (except count day). The last gives the sum of the count days and count periods reporting each species. 
Table 1.32 ${ }^{\text {nd }}$ Saskatchewan Christmas Mammal Count-2004.

Short-tailed Shrew

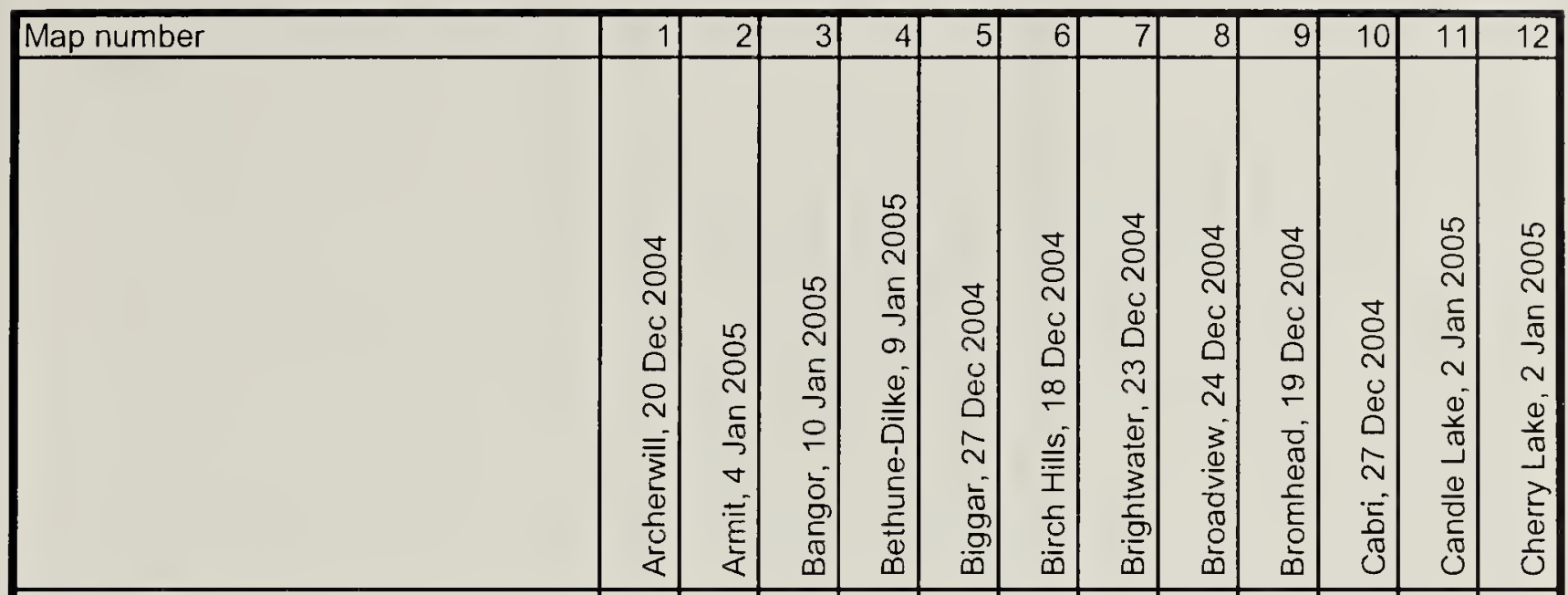

Shrew Species

Nuttall's Cottontail

Snowshoe Hare

White-tailed Jack Fabbit

Least Chipmunk

Eastern Grey Squirrel

Fox Squirrel

American Red Squirrel

Northern Flying-Squirrel

American Beaver

Deer Mouse

Muskrat

Gapper's Red-Backed Vole

Meadow Vole

Vole Species

Mouse Species

Norway Rat

House Mouse

American Porcupine

Coyote

Wolf

Red Fox

Swift Fox

Raccoon

American Marten

Fisher

Ermine

Long-tailed Weasel

Least Weasel

Weasel Species

American Mink

American Badger

Striped Skunk

River Otter

Lynx

Woodland Caribou

Mule Deer

White-tailed Deer

\begin{tabular}{|r|r|r|r|r|r|r|r|r|}
\hline \\
\hline
\end{tabular}

Deer Species

Moose

Elk

Pronghorn

\begin{tabular}{r|r|r|r|r|r|r|r|r|r}
\hline $\mathrm{t}$ & & 9 & & $\mathrm{t}$ & & & & & 1 \\
\hline
\end{tabular}

Totals seen/heard on count day

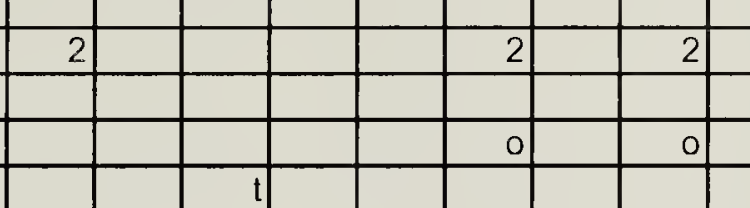

1

1

Total species seen/heard

Total species recorded by tracks

Total species otherwise recorded

\begin{tabular}{lll|l|l|l|l|l}
\hline & & $\mathrm{t}$ & & & \\
\hline
\end{tabular}

Species recorded count period

Total species count period and day 
Table 1. $32^{\text {nd }}$ Saskatchewan Christmas Mammal Count-2004.

\begin{tabular}{|c|c|c|c|c|c|c|c|c|c|c|c|c|}
\hline Map number & 14 & 15 & 16 & 17 & 18 & 19 & 20 & \begin{tabular}{l|l}
21 \\
\end{tabular} & 22 & 23 & 24 & 25 \\
\hline & 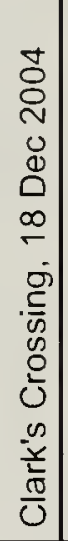 & 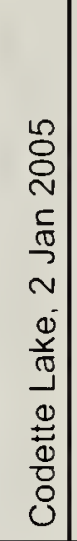 & 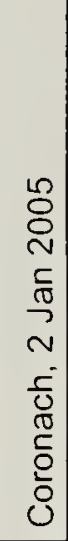 & 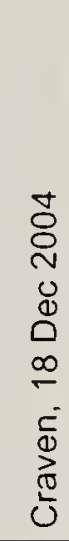 & $\begin{array}{l}0 \\
0 \\
N \\
0 \\
d \\
0 \\
N \\
0 \\
0 \\
\frac{1}{0} \\
د \\
0 \\
0 \\
0 \\
0 \\
0\end{array}$ & 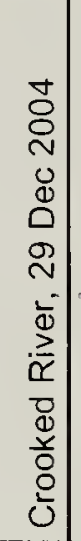 & 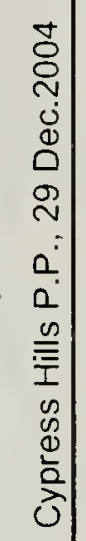 & 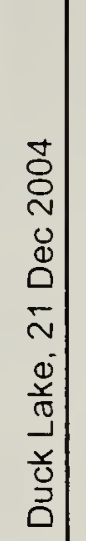 & $\begin{array}{c}10 \\
0 \\
0 \\
N \\
\frac{1}{D} \\
0 \\
5 \\
5 \\
\frac{5}{0} \\
5\end{array}$ & 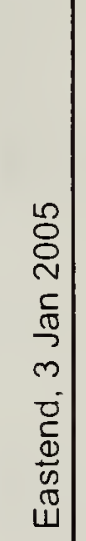 & 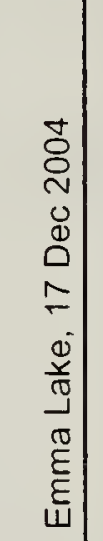 & 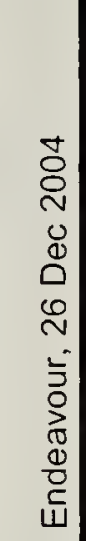 \\
\hline \multicolumn{13}{|l|}{ Short-tailed Shrew } \\
\hline \multicolumn{13}{|l|}{ Shrew Species } \\
\hline Nuttall's Cottontail & & & & & & & 2 & & & 2 & & \\
\hline Snowshoe Hare & 4 & $t_{1}$ & & & & & 1 & it & t & t & $\mathrm{t}$ & $t$ \\
\hline White-tailed Jack Rabbit & & & 2 & & & & & & $\mathrm{t}$ & 23 & & \\
\hline \multicolumn{13}{|l|}{ Least Chipmunk } \\
\hline Eastern Grey Squirrel & & & & 4 & & & & & & & & \\
\hline \multicolumn{13}{|l|}{ Fox Squirrel } \\
\hline American Red Squirrel & 1 & 2 & & 5 & & 1 & 3 & 6 & $\mathrm{t}$ & & 5 & 3 \\
\hline \multicolumn{13}{|l|}{ Northern Flying-Squirrel } \\
\hline American Beaver & & & & & of & & & & & & & \\
\hline \multicolumn{13}{|l|}{ Deer Mouse } \\
\hline \multicolumn{13}{|l|}{ Muskrat } \\
\hline \multicolumn{13}{|l|}{ Gapper's Red-Backed Vole } \\
\hline \multicolumn{13}{|l|}{ Meadow Vole } \\
\hline \multirow{2}{*}{\multicolumn{13}{|c|}{ Vole Species }} \\
\hline \multicolumn{6}{|l|}{ Mouse Species } & & & & & & & \\
\hline \multicolumn{13}{|l|}{ Norway Rat } \\
\hline \multicolumn{13}{|l|}{ House Mouse } \\
\hline American Porcupine & & $t$ & & $\mathrm{t}$ & & & & & & & & $\mathrm{t}$ \\
\hline \multicolumn{13}{|l|}{ Coyote } \\
\hline \multicolumn{13}{|l|}{\begin{tabular}{|l|l} 
Wolf \\
\end{tabular}} \\
\hline Red Fox & 3 & $\mathrm{t}$ & 1 & 1 & & & & & & & & \\
\hline \multicolumn{13}{|l|}{ Swift Fox } \\
\hline \multicolumn{13}{|l|}{ Raccoon } \\
\hline American Marten & & & & & & & & & & & & \\
\hline Fisher & & & & & & & & & & & & \\
\hline Ermine & & & & & & & & & & & & \\
\hline \begin{tabular}{|l} 
Long-tailed Weasel \\
\end{tabular} & & $\mathrm{t}$ & & & & & 3 & & & & & \\
\hline Least Weasel & & & & & & & $t$ & & & $\mathrm{t}$ & & \\
\hline Weasel Species & $t$ & & & & & & & & & & & \\
\hline American Mink & & & & & & & & & & & & \\
\hline American Badger & & & 0 & & & & & & & & & \\
\hline Striped Skunk & & & & & & & & & & & & \\
\hline River Otter & & & & & & & & & & & & \\
\hline $\operatorname{Lyn} x$ & & & & & & & $\mathrm{t}$ & & & & & \\
\hline Woodland Caribou & & & & & & & & & & & & \\
\hline Mule Deer & 12 & t & 13 & 1 & & & 12 & & & & & \\
\hline White-tailed Deer & 12 & t & 7 & 9 & $t$ & 4 & 3 & \begin{tabular}{l|l}
3 & \\
\end{tabular} & 1 & 5 & t & 5 \\
\hline Deer Species & & & & & & & 12 & & & & & \\
\hline Moose & & & & & & & 3 & & & 2 & & $\mathrm{t}$ \\
\hline Elk & & 85 & & & & & & & & & & \\
\hline Pronghorn & & & & & & & & & & 9 & & \\
\hline 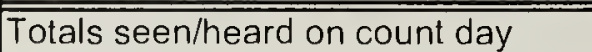 & (33 & 88 & 24 & $\overline{27}$ & 0 & (5) & 39 & 10 & 1 & (43) & 5 & $\overline{11}$ \\
\hline Total species seen/heard & 6 & 3 & 5 & 7 & 0 & 2 & 7 & $3 \mid$ & 1 & 6 & 1 & 5 \\
\hline Total species recorded by tracks & 1 & 6 & 1 & 1 & 2 & 0 & 4 & 2 & 6 & 1 & 2 & 5 \\
\hline Total species otherwise recorded & 0 & 0 & 1 & 0 & 1 & 0 & 0 & 0 & 0 & 0 & 0 & 0 \\
\hline Species recorded count period & 0 & 0 & 0 & 0 & 0 & 0 & 0 & 0 & 0 & 0 & 0 & 0 \\
\hline Total species count period and day & 7 & 9 & 7 & 8 & 3 & 2 & 11 & 5 & 7 & 7 & 3 & 10 \\
\hline
\end{tabular}


Table 1. 32 ${ }^{\text {nd }}$ Saskatchewan Christmas Mammal Count-2004.

\begin{tabular}{|c|c|c|c|c|c|c|c|c|c|c|c|c|}
\hline Map number & 26 & 27 & 28 & 29 & 30 & 31 & 32 & 33 & 34 & 35 & 37 & 38 \\
\hline & 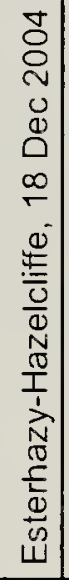 & 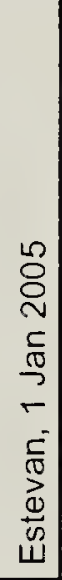 & 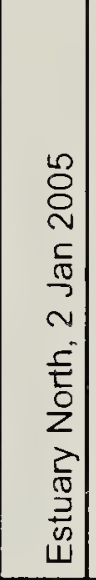 & 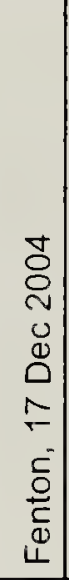 & 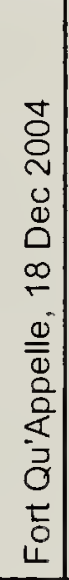 & 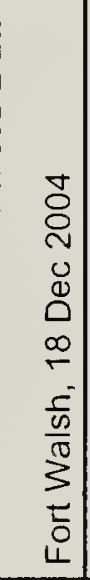 & 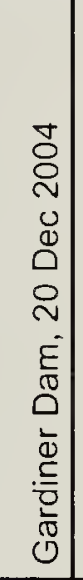 & 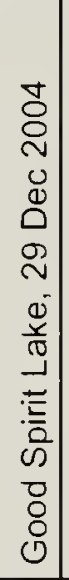 & $\begin{array}{l} \pm \\
0 \\
0 \\
0 \\
0 \\
0 \\
0 \\
\square \\
\vdots \\
0 \\
0 \\
0 \\
0 \\
0 \\
0 \\
0\end{array}$ & 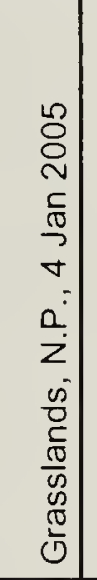 & 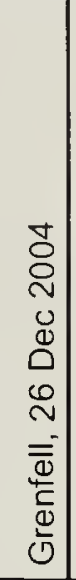 & 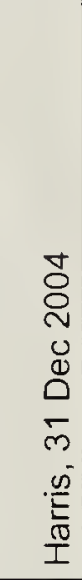 \\
\hline \multicolumn{13}{|l|}{ Short-tailed Shrew } \\
\hline \multicolumn{13}{|l|}{ Shrew Species } \\
\hline Nuttall's Cottontail & & & 8 & & & t) & 4 & & 2 & 1 & & \\
\hline Snowshoe Hare & & & & $\mathrm{t}$ & & & $\mathrm{t}$ & $\mathrm{t}$ & - & - & & \\
\hline White-tailed Jack Rabbit & & & 2 & & & 2 & 1 & $\mathrm{t}$ & 3 & 2 & $\mathrm{t}$ & 1 \\
\hline \multicolumn{13}{|l|}{ Least Chipmunk } \\
\hline \multicolumn{13}{|l|}{ Eastern Grey Squirrel } \\
\hline \multicolumn{13}{|l|}{ Fox Squirrel } \\
\hline American Red Squirrel & 1 & & & 8 & 12 & 25 & & 3 & & & & \\
\hline \multicolumn{13}{|l|}{ Northern Flying-Squirrel } \\
\hline American Beaver & & & & 7 & c & & & o & & & & \\
\hline Deer Mouse & & & & & 3 & & & 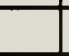 & & & & \\
\hline Muskrat & & & & & 1 & & & o & & & & \\
\hline \multicolumn{13}{|l|}{ Gapper's Red-Backed Vole } \\
\hline \multicolumn{13}{|l|}{ Meadow Vole } \\
\hline \multicolumn{13}{|l|}{ Vole Species } \\
\hline \multicolumn{13}{|l|}{ Mouse Species } \\
\hline \multicolumn{13}{|l|}{ Norway Rat } \\
\hline \multicolumn{13}{|l|}{ House Mouse } \\
\hline American Porcupine & & & & & & 2 & & $\mathrm{t}$ & & & & \\
\hline Coyote & & & 2 & 3 & 1 & & 1 & 2 & 5 & 12 & & \\
\hline \multicolumn{13}{|l|}{ Wolf } \\
\hline Red Fox & & & & & c & & & $\mathrm{t}$ & 1 & of & & \\
\hline Swift Fox & & & & & & & & 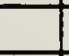 & & of & & \\
\hline \multicolumn{13}{|l|}{ Raccoon } \\
\hline \multicolumn{13}{|l|}{ American Marten } \\
\hline \multicolumn{13}{|l|}{ Fisher } \\
\hline \multicolumn{13}{|l|}{ Ermine } \\
\hline Long-tailed Weasel & & & & & & & & & 1 & & & \\
\hline Least Weasel & & & & & & & & & & & & \\
\hline Weasel Species & & & & & & & & & & & & \\
\hline American Mink & & & & & & & & $\mathrm{t}$ & & & & \\
\hline American Badger & & & & & & & & 1 & & & & \\
\hline Striped Skunk & & & & & & & & & & & & \\
\hline River Otter & & & & & & & & & & & & \\
\hline Lynx & & & & & & & & & & & & \\
\hline Woodland Caribou & & & & & & & & & & & & \\
\hline Mule Deer & & 2 & 111 & & c & 39 & 33 & & 112 & 117 & & 141 \\
\hline White-tailed Deer & & 2 & 150 & 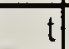 & 5 & 39 & 35 & 6 & 3 & 142 & & 16 \\
\hline Deer Species & & & & & & & & & & & & \\
\hline Moose & & & & & & & & & & & - & \\
\hline Elk & & & & & & $\mathrm{t}$ & & $\mathrm{t}$ & & & & \\
\hline Pronghorn & & & & & & & 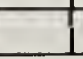 & $t$ & 69 & 84 & & 17 \\
\hline Totals seen/heard on count day & 1 & 5 & 273 & 18 & 25 & 108 & 74 & 12 & 196 & 358 & 0 & 175 \\
\hline Total species seen/heard & 1 & 3 & 5 & 3 & 8 & 6 & 5 & 4 & 8 & 6 & 0 & 4 \\
\hline Total species recorded by tracks & 0 & 0 & 0 & 3 & 0 & 2 & 1 & 9 & 0 & 1 & 1 & 1 \\
\hline Total species otherwise recorded & 0 & 0 & 0 & 0 & 0 & 0 & 0 & 2 & 0 & 2 & 0 & 0 \\
\hline Species recorded count period & 0 & 0 & 0 & 0 & 3 & 0 & 0 & 0 & 0 & 0 & 0 & 0 \\
\hline Total species count period and day & 1 & 3 & 5 & 6 & 11 & 8 & 6 & 15 & 8 & 9 & 1 & 5 \\
\hline
\end{tabular}


Table 1. 32 ${ }^{\text {nd }}$ Saskatchewan Christmas Mammal Count-2004.

\begin{tabular}{|c|c|c|c|c|c|c|c|c|c|c|c|c|}
\hline Map number & 39 & 41 & 42 & 43 & 44 & 45 & 46 & 47 & 48 & 49 & 50 & 51 \\
\hline & 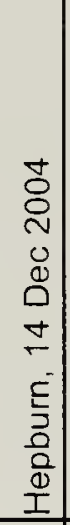 & 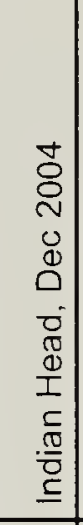 & 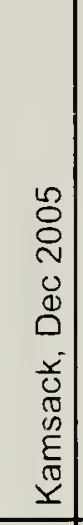 & 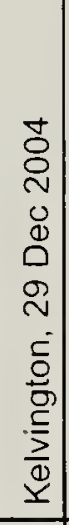 & 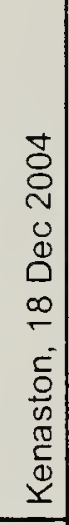 & $\begin{array}{c} \pm \\
0 \\
0 \\
0 \\
0 \\
0 \\
0 \\
N \\
0 \\
0 \\
\frac{0}{\pi} \\
1 \\
0 \\
0 \\
0 \\
0 \\
0 \\
1 \\
\end{array}$ & 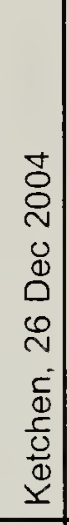 & 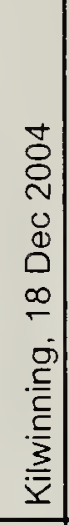 & 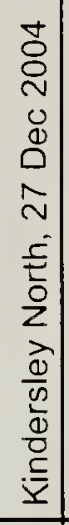 & 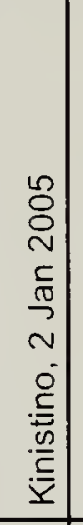 & 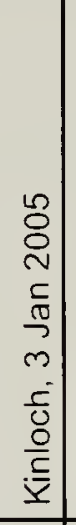 & 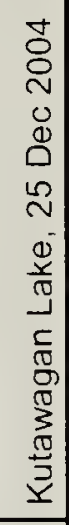 \\
\hline \multicolumn{13}{|l|}{ Short-tailed Shrew } \\
\hline \multicolumn{13}{|l|}{ Shrew Species } \\
\hline \multicolumn{13}{|l|}{ Nuttall's Cottontail } \\
\hline Snowshoe Hare & & 27 & & $\mathrm{t}$ & & & & & & & $\mathrm{t}$ & \\
\hline White-tailed Jack Rabbit & & 9 & & $t$ & $t$ & & & & & & & \\
\hline \multicolumn{13}{|l|}{ Least Chipmunk } \\
\hline \multicolumn{13}{|l|}{ Eastern Grey Squirrel } \\
\hline Fox Squirrel & & 2 & & & & & & & & & & \\
\hline American Red Squirrel & & 34 & 3 & 1 & & 11 & & 8 & & 2 & 3 & \\
\hline \multicolumn{13}{|l|}{ Northern Flying-Squirrel } \\
\hline American Beaver & & & & & 0 & 0 & & & & & of & \\
\hline \multicolumn{13}{|l|}{ Deer Mouse } \\
\hline Muskrat & & & & & $\mathrm{O}$ & & & & & & o & \\
\hline \multicolumn{13}{|l|}{ Gapper's Red-Backed Vole } \\
\hline \multicolumn{13}{|l|}{ Meadow Vole } \\
\hline \multicolumn{13}{|l|}{ Vole Species } \\
\hline \multicolumn{13}{|l|}{ Mouse Species } \\
\hline \multicolumn{13}{|l|}{ Norway Rat } \\
\hline \multicolumn{13}{|l|}{ House Mouse } \\
\hline American Porcupine & & 3 & & & $\mathrm{t}$ & $t$ & & & & 3 & & 1 \\
\hline Coyote & & 15 & 12 & $t$ & $\mathrm{t}$ & 3 & & 1 & $\mathrm{c}$ & 2 & t & \\
\hline Wolf & & & 2 & & 7 & 4 & & & 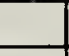 & 1 & & \\
\hline Red Fox & & 5 & 6 & $\mathrm{t}$ & $\mathrm{t}$ & $\mathrm{t}$ & & & $c$ & 1 & & \\
\hline \multicolumn{13}{|l|}{ Swift Fox } \\
\hline Raccoon & & 1 & & & $\mathrm{t}$ & & & & & 2 & & \\
\hline \multicolumn{13}{|l|}{ American Marten } \\
\hline \multicolumn{13}{|l|}{ Fisher } \\
\hline \multicolumn{13}{|l|}{ Ermine } \\
\hline Long-tailed Weasel & & 3 & & & $\mathrm{t}$ & & & & & & & \\
\hline Least Weasel & & & & & & & & & & & $\mathrm{t}$ & \\
\hline Weasel Species & & 2 & & & & & & & & 1 & & $t$ \\
\hline American Mink & & & & & & & & & & & & \\
\hline American Badger & & & & & & & & & & & & \\
\hline Striped Skunk & & & & 0 & $\mathrm{t}$ & & & & & & & \\
\hline River Otter & & & & & & & & & & & & \\
\hline Lynx & & & 1 & & & & & & & & & \\
\hline Woodland Caribou & & & & & & & & & & & & \\
\hline Mule Deer & & 13 & 5 & & 26 & & & & 2 & & & 1 \\
\hline White-tailed Deer & & 48 & 154 & $t$ & 5 & 12 & 10 & 4 & & & 3 & 8 \\
\hline Deer Species & & & 4 & & & & & & & & & \\
\hline Moose & & 1 & 4 & $\mathrm{C}$ & $t$ & 1 & & & & & $t$ & \\
\hline Elk & & & 8 & & & $t$ & $\mathrm{c}$ & & & & $\mathrm{t}$ & \\
\hline Pronghorn & & & & & $t$ & & & & & & & \\
\hline Totals seen/heard on count day & 0 & 163 & 201 & 2 & 31 & 26 & 11 & 13 & 2 & 11 & 6 & 10 \\
\hline Total species seen/heard & 0 & 12 & 10 & 2 & 2 & 3 & 2 & 3 & 1 & 6 & 2 & 3 \\
\hline Total species recorded by tracks & 1. & 1 & 0 & 7 & 11 & 5 & 0 & 0 & 0 & 1 & 6 & 1 \\
\hline Total species otherwise recorded & 0 & 0 & 0 & 1 & 2 & 1 & 0 & 0 & 0 & 0 & 2 & 0 \\
\hline Species recorded count period & 0 & 0 & 0 & 1 & 0 & 0 & 1 & 0 & 2 & 0 & 0 & 0 \\
\hline Total species count period and day & 1 & 13 & 10 & 11 & 15 & 9 & 3 & 3 & 3 & 7 & 10 & 4 \\
\hline
\end{tabular}


Table 1. 32 $2^{\text {nd }}$ Saskatchewan Christmas Mammal Count-2004.

\begin{tabular}{|c|c|c|c|c|c|c|c|c|c|c|c|c|}
\hline \multirow[t]{2}{*}{ Map number } & 52 & 53 & 54 & 55 & 56 & 57 & 58 & 59 & 60 & 61 & 62 & 63 \\
\hline & 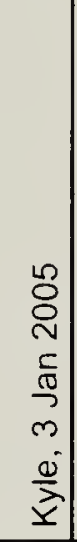 & 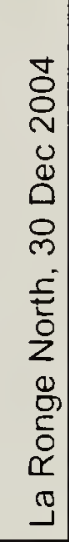 & 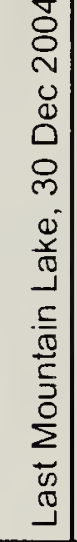 & 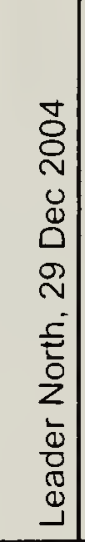 & 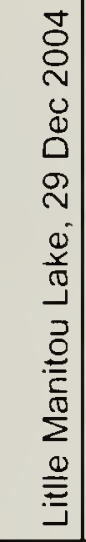 & 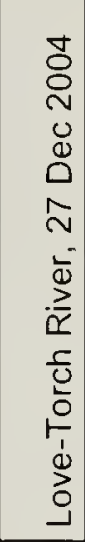 & 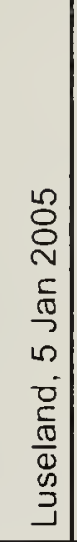 & 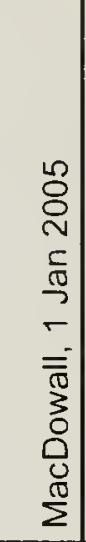 & 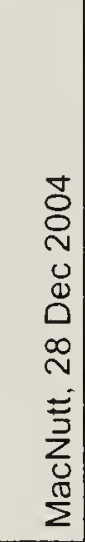 & $\begin{array}{l}0 \\
0 \\
N \\
0 \\
0 \\
0 \\
0 \\
N \\
0 \\
0 \\
0 \\
\frac{\pi}{\pi} \\
\Sigma\end{array}$ & 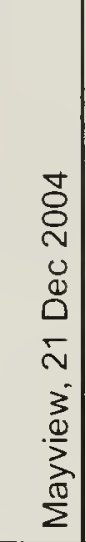 & 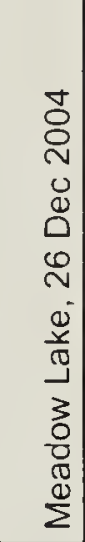 \\
\hline \multicolumn{13}{|l|}{ Short-tailed Shrew } \\
\hline \multicolumn{13}{|l|}{ Shrew Species } \\
\hline \multicolumn{13}{|l|}{ Nuttall's Cottontail } \\
\hline \multicolumn{13}{|l|}{ Snowshoe Hare } \\
\hline \multicolumn{13}{|l|}{ White-tailed Jack Rabbit } \\
\hline \multirow{2}{*}{\multicolumn{13}{|c|}{ Least Chipmunk }} \\
\hline \multicolumn{7}{|l|}{ Eastern Grey Squirrel } & & & & & & \\
\hline \multicolumn{13}{|l|}{ Fox Squirrel } \\
\hline American Red Squirrel & & 3 & & & & 3 & & 2 & 3 & & 3 & \\
\hline \multicolumn{13}{|l|}{ Northern Flying-Squirrel } \\
\hline \multicolumn{13}{|l|}{ American Beaver } \\
\hline \multicolumn{13}{|l|}{ Deer Mouse } \\
\hline \multicolumn{13}{|l|}{ Muskrat } \\
\hline \multirow{2}{*}{\multicolumn{13}{|c|}{ Gapper's Red-Backed Vole }} \\
\hline \multirow{2}{*}{\multicolumn{13}{|c|}{$\frac{\text { Meadow Vole }}{\text { Vole Snecies }}$}} \\
\hline & & & & & & & & & & & & \\
\hline \multicolumn{13}{|l|}{ Mouse Species } \\
\hline \multicolumn{13}{|l|}{ Norway Rat } \\
\hline House Mouse & & & & & & & & & & & & \\
\hline American Porcupine & 1 & & & & $\mathrm{o}$ & & & $\mathrm{c}$ & & 1 & & \\
\hline Coyote & 5 & & t & 1 & $t$ & 1 & 4 & 2 & 2 & 6 & 1 & 3 \\
\hline Wolf & & & & & & & & & & & & \\
\hline Red Fox & $\mathrm{t}$ & & & & & & c| & $\mathrm{t}$ & & & & $l$ \\
\hline Swift Fox & & & & & & & & & & & & \\
\hline Raccoon & & & & & & $\mathrm{t}$ & & & & & & \\
\hline American Marten & & & & & & & & & & & & \\
\hline Fisher & & & & & & & & & & & & 6 \\
\hline Ermine & & & & & & & & $\mathrm{t}$ & & & & \\
\hline Long-tailed Weasel & & & & & & & & & & & & \\
\hline Least Weasel & & & & 1 & & & & & & & & \\
\hline Weasel Species & $\mathrm{t}$ & & $\mathrm{t}$ & & & $\mathrm{c}$ & & & & & & $\mathrm{t}$ \\
\hline American Mink & & & & & & $\mathrm{c}$ & & & & & & \\
\hline American Badger & & & & & & & & 0 & & & & \\
\hline Striped Skunk & & & & & $\mathrm{o}$ & & & & & & & \\
\hline River Otter & & & & & & & & & & & & \\
\hline Lynx & & 1 & & & & & & $t$ & & & & \\
\hline Woodland Caribou & & & & & & & & & & & & \\
\hline Mule Deer & 12 & & 2 & 11 & 3 & & 11 & & & 51 & & \\
\hline White-tailed Deer & 40 & & 24 & 10 & 1 & 43 & & 6 & $t$ & 50 & & 5 \\
\hline Deer Species & & & & & & & & & & & & \\
\hline Moose & & & & & & $\mathrm{t}$ & $\mathrm{c}$ & & & & & \\
\hline Elk & & & & & & & & & & & 3 & \\
\hline Pronghorn & & & & & & & & & & & & \\
\hline Totals seen/heard on count day & 71 & 4 & 27 & 33 & 4 & 47 & 19 & 10 & 5 & \begin{tabular}{|c|}
110 \\
\end{tabular} & 7 & 8 \\
\hline Total species seen/heard & 7 & 2 & 3 & 7 & 2 & 3 & 3 & 3 & 2 & 6 & 3 & 2 \\
\hline Total species recorded by tracks & 2 & 0 & 2 & 0 & 2 & 3 & 0 & 4 & 1 & 2 & 0 & 6 \\
\hline Total species otherwise recorded & 0 & 0 & 0 & 0 & 2 & 0 & 0 & 2 & 0 & 0 & 0 & 0 \\
\hline Species recorded count period & 0 & 0 & 0 & 0 & 1 & 2 & 3 & 2 & 0 & 0 & 0 & 0 \\
\hline Total species count period and day & 9 & 2 & 5 & 7 & 7 & 8 & 6 & 11 & 3 & 8 & 3 & 8 \\
\hline
\end{tabular}


Table 1. 32 ${ }^{\text {nd }}$ Saskatchewan Christmas Mammal Count-2004.

\begin{tabular}{|c|c|c|c|c|c|c|c|c|c|c|c|c|}
\hline Map number & 64 & 65 & 66 & 67 & 68 & 69 & 70 & 71 & 72 & 73 & 74 & 75 \\
\hline & 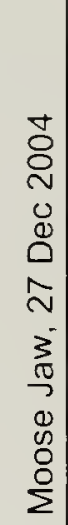 & 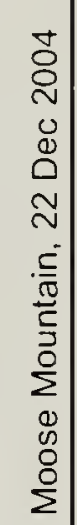 & 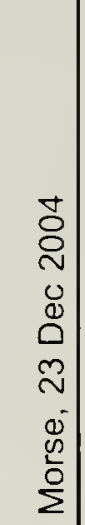 & 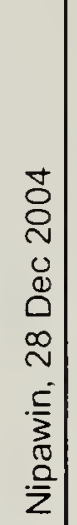 & 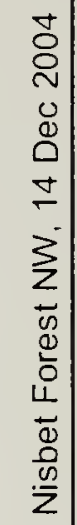 & 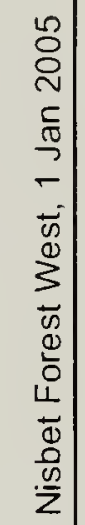 & 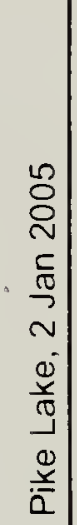 & 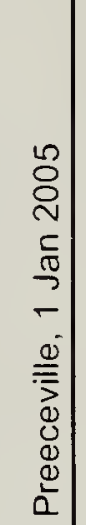 & 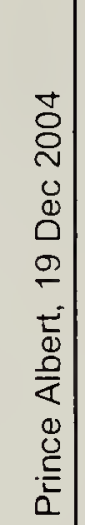 & 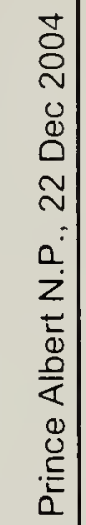 & 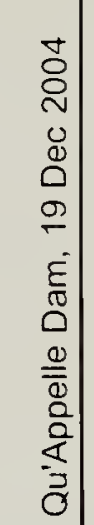 & 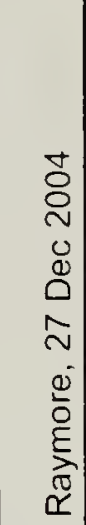 \\
\hline \multicolumn{13}{|l|}{ Short-tailed Shrew } \\
\hline \multicolumn{13}{|l|}{ Shrew Species } \\
\hline Nuttall's Cottontail & $\mathrm{t}$ & & $t$ & & & & & & & & & \\
\hline Snowshoe Hare & 2 & & & 10 & c & & 2 & & 5 & 1 & & \\
\hline While-tailed Jack Rabbit & 2 & & $t$ & 2 & & & 1 & & $\mathrm{t}$ & & & \\
\hline \multicolumn{13}{|l|}{ Least Chipmunk } \\
\hline \multicolumn{13}{|l|}{ Eastern Grey Squirrel } \\
\hline \multicolumn{13}{|l|}{ Fox Squirrel } \\
\hline American Red Squirrel & & 3 & & 5 & $\mathrm{c}$ & 7 & 4 & 4 & 8 & $\mathrm{t}$ & & \\
\hline \multicolumn{13}{|l|}{ Northern Flying-Squirrel } \\
\hline American Beaver & & & & & & & & & 0 & & & \\
\hline Deer Mouse & & & & & & & & & $\mathrm{t}$ & & & \\
\hline \multicolumn{13}{|l|}{ Muskrat } \\
\hline \multicolumn{13}{|l|}{ Gapper's Red-Backed Vole } \\
\hline \multicolumn{13}{|l|}{ Meadow Vole } \\
\hline Vole Species & & & 1 & & & & & & 2 & & & \\
\hline \multicolumn{13}{|l|}{ Mouse Species } \\
\hline \multicolumn{13}{|l|}{ Norway Rat } \\
\hline \multicolumn{13}{|l|}{ House Mouse } \\
\hline \multicolumn{13}{|l|}{ American Porcupine } \\
\hline Coyote & 1 & 1 & 4 & 1 & 1 & & 4 & & 3 & $\mathrm{t}$ & 2 & \\
\hline \multicolumn{13}{|l|}{ Wolf } \\
\hline \multicolumn{13}{|l|}{\begin{tabular}{|l} 
Red Fox \\
Swift Foxx
\end{tabular}} \\
\hline Swift Fox & & & & & & & & & & & & \\
\hline \multicolumn{13}{|l|}{ Raccoon } \\
\hline \multicolumn{13}{|l|}{ American Marten } \\
\hline Fisher & & & & & & & & & & $\mathrm{t}$ & & \\
\hline Ermine & & & & & & 1 & & & & & & \\
\hline \begin{tabular}{|l} 
Long-tailed Weasel \\
\end{tabular} & & & & 1 & & & & & $\mathrm{t}$ & & & \\
\hline Least Weasel & & & & & & & & & & & & \\
\hline Weasel Species & & & & & & & & & & $t$ & & \\
\hline American Mink & & & & & & & & & $\mathrm{t}$ & & & \\
\hline American Badger & & & of & & & & & & & & & \\
\hline Striped Skunk & & & & & $\mathrm{c}$ & & & & & & & \\
\hline River Otter & & & & & & & & & & 2 & & \\
\hline Lynx & & & & & & & & & & & & \\
\hline Woodland Caribou & & & & & & & & & & & & \\
\hline Mule Deer & & & 24 & & & & 2 & & & & 50 & \\
\hline White-tailed Deer & & 7 & 33 & 15 & $\mathrm{c}$ & & 35 & 8 & $\mathrm{t}$ & 1 & 24 & 22 \\
\hline Deer Species & & & & & & & & & & & & \\
\hline Moose & & & 3 & c & & & & & & & & \\
\hline Elk & & & & & & & & & & $\mathrm{t}$ & & \\
\hline Pronghorn & & & & & & & & & & & 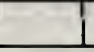 & \\
\hline Totals seen/heard on count day & 9 & 11 & 65 & 36 & 1 & 8 & 49 & 13 & 18 & 3 & $77 \mid$ & 22 \\
\hline Total species seen/heard & 4 & 3 & 5 & 7 & 1 & 2 & 7 & $3 \mid$ & 4 & 2 & 4 & 1 \\
\hline Total species recorded by tracks & 2 & 0 & 2 & 0 & 0 & 0 & 1 & 0 & 5 & 10 & 0 & 1 \\
\hline Total species otherwise recorded & 0 & 0 & 1 & 0 & 1 & 0 & 0 & 0 & 1 & 0 & 0 & $\overline{0}$ \\
\hline Species recorded count period & 0 & 0 & 1 & 1 & 5 & 1 & 0 & 0 & 0 & 0 & 0 & 0 \\
\hline Total species count period and day & 6 & 3 & 9 & 8 & 7 & 3 & 8 & 3 & 10 & 12 & 4 & 2 \\
\hline
\end{tabular}


Table 1. $32^{\text {nd }}$ Saskatchewan Christmas Mammal Count-2004.

\begin{tabular}{|c|c|c|c|c|c|c|c|c|c|c|c|c|}
\hline Map number & 76 & 78 & 79 & 80 & 81 & 82 & 83 & 84 & 85 & 86 & 87 & 88 \\
\hline & 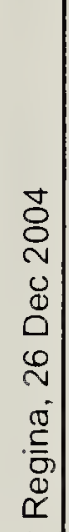 & 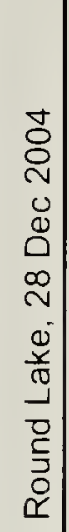 & 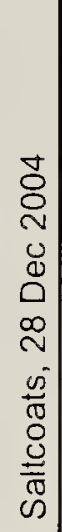 & 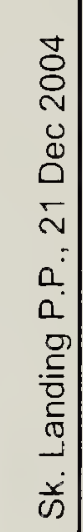 & 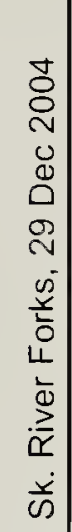 & 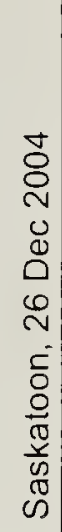 & 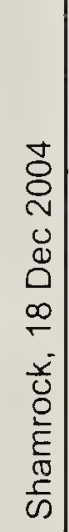 & 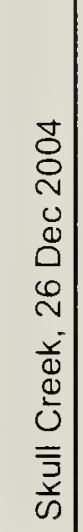 & 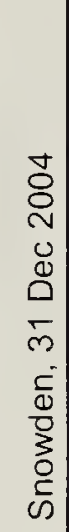 & 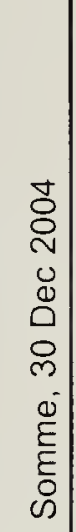 & 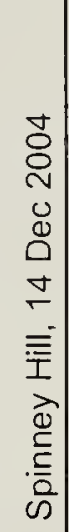 & 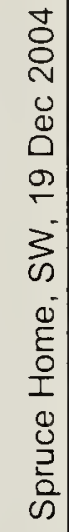 \\
\hline \multicolumn{13}{|l|}{ Short-tailed Shrew } \\
\hline Shrew Species & & & & & & & & & & & $\mathrm{t}$ & \\
\hline Nuttall's Cottontail & & & & 10 & & & & 4 & & & & \\
\hline Snowshoe Hare & $\mathrm{t}$ & 1 & 1 & & $\mathrm{t}$ & 3 & & 4 & 9 & & $\mathrm{t}$ & \\
\hline White-tailed Jack Rabbit & 24 & $\mathrm{t}$ & & & & 14 & & 2 & 1 & 1 & $t$ & \\
\hline \multicolumn{13}{|l|}{ Least Chipmunk } \\
\hline Eastern Grey Squirrel & 2 & & & & & & & & & & & \\
\hline Fox Squirrel & 4 & & & & & & & & & & & \\
\hline American Red Squirrel & 8 & 4 & 2 & & 7 & & & 2 & 10 & 8 & 9 & 2 \\
\hline \multicolumn{13}{|l|}{ Northern Flying-Squirrel } \\
\hline American Beaver & 암 & 0 & & & & 3 & & & & & & \\
\hline Deer Mouse & $\mathrm{t}$ & & & $\mathrm{t}$ & & & & & & $c$ & $\mathrm{t}$ & \\
\hline \multicolumn{13}{|l|}{ Muskrat } \\
\hline \multicolumn{13}{|l|}{ Gapper's Red-Backed Vole } \\
\hline Meadow Vole & $\mathrm{t}$ & 1 & & & 1 & $\mathrm{t}$ & & & $\mathrm{t}$ & & & \\
\hline \multicolumn{13}{|l|}{ Vole Species } \\
\hline \multicolumn{13}{|l|}{ Mouse Species } \\
\hline \multicolumn{13}{|l|}{ Norway Rat } \\
\hline \multicolumn{13}{|l|}{ House Mouse } \\
\hline American Porcupine & & & 0 & 2 & $\mathrm{t}$ & 1 & & & & & & \\
\hline Coyote & 5 & 4 & 1 & 7 & $\mathrm{t}$ & 3 & 1. & 4 & 1 & 1 & 2 & $c$ \\
\hline \multicolumn{13}{|l|}{ Wolf } \\
\hline Red Fox & 1 & $\mathrm{t}$ & & 2 & 1 & 1 & & 1 & & 1 & & $\mathrm{C}$ \\
\hline \multicolumn{13}{|l|}{ Swift Fox } \\
\hline \multicolumn{13}{|l|}{ Raccoon } \\
\hline \multicolumn{13}{|l|}{ American Marten } \\
\hline Fisher & & & & & & & & & & & 1 & \\
\hline Ermine & & & & & & 1 & & & & & & \\
\hline \multicolumn{13}{|l|}{ Long-tailed Weasel } \\
\hline \multicolumn{13}{|l|}{ Least Weasel } \\
\hline Weasel Species & & & & $\mathrm{t}$ & & & & & & & & $\mathrm{c}$ \\
\hline \multicolumn{13}{|l|}{ American Mink } \\
\hline American Badger & & & & & & & o & 1 & & & & \\
\hline \multicolumn{13}{|l|}{ Striped Skunk } \\
\hline River Otter & & & & & & & & & & & & \\
\hline $\operatorname{Lyn} x$ & & & & & & & & & & & & \\
\hline Woodland Caribou & & & & & & & & & & & & \\
\hline Mule Deer & 16 & $\mathrm{t}$ & & 52 & & 4 & 2 & 55 & & c & 1 & \\
\hline White-tailed Deer & 3 & $\mathrm{t}$ & 5 & 15 & $\mathrm{t}$ & 54 & 4 & 75 & 8 & 25 & 4 & \\
\hline Deer Species & & & & & & & 34 & & & & & \\
\hline Moose & & & & & & & & & $\mathrm{t}$ & 1 & & \\
\hline Elk & & & & & $\mathrm{t}$ & & & & & 10 & & \\
\hline Pronghorn & & & & 34 & & & & & & & & \\
\hline Totals seen/heard on count day & 63 & 10 & 9 & 122 & 9 & 84 & 41 & 146 & 29 & 47 & 17 & 2 \\
\hline Total species seen/heard & 8 & 4 & 4 & 7 & 3 & 9 & 3 & 9 & 5 & 7 & 5 & 1 \\
\hline Total species recorded by tracks & 3 & 4 & 0 & 3 & 6 & 1 & 0 & 0 & 5 & 0 & 4 & 0 \\
\hline Total species otherwise recorded & 1 & 1 & 2 & 0 & 0 & 0 & 1 & 0 & 0 & 0 & 0 & 0 \\
\hline Species recorded count period & 0 & 0 & 0 & 0 & 0 & 0 & 0 & 0 & 1 & 3 & 0 & 3 \\
\hline Total species count period and day & 12 & 9 & 6 & 10 & 9 & 10 & 4 & 9 & 11 & 10 & 9 & 4 \\
\hline
\end{tabular}


Table 1. 32 $2^{\text {nd }}$ Saskatchewan Christmas Mammal Count-2004.

\begin{tabular}{|c|c|c|c|c|c|c|c|c|c|c|c|}
\hline Map number & 89 & 90 & 91 & 92 & 93 & 94 & 95 & 96 & 97 & 98 & 99 \\
\hline & 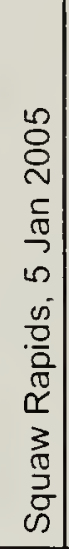 & 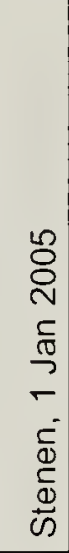 & 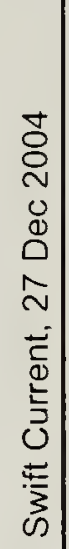 & 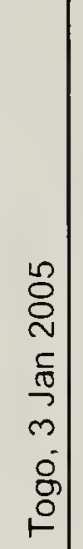 & 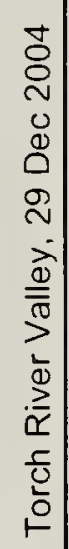 & 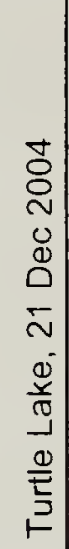 & 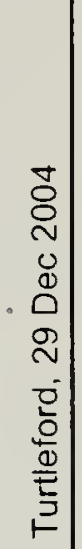 & 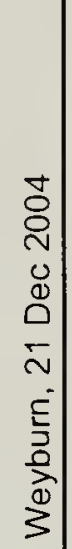 & 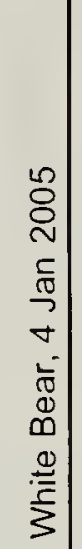 & 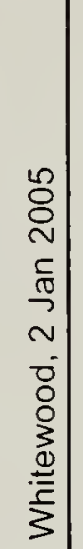 & 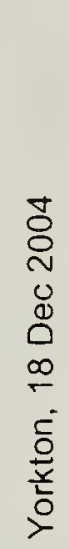 \\
\hline \multicolumn{12}{|l|}{ Short-tailed Shrew } \\
\hline \multicolumn{12}{|l|}{ Shrew Species } \\
\hline Nuttall's Cottontail & & & 2 & & & & & & 1 & & \\
\hline Snowshoe Hare & $\mathrm{t}$ & & & 3 & & 2 & 1 & & & $\mathrm{t}$ & 1 \\
\hline White-tailed Jack Rabbit & & & 8 & & & 1 & & 2 & & $\mathrm{t}$ & $\overline{1}$ \\
\hline \multicolumn{12}{|l|}{ Least Chipmunk } \\
\hline Eastern Grey Squirrel & & & 16 & & & & & & & $\mathrm{c}$ & \\
\hline Fox Squirrel & & & & & & & & 4 & & $\mathrm{c}$ & \\
\hline American Red Squirrel & & 6 & & 4 & 11 & 3 & 6 & & & 2 & 3 \\
\hline Northern Flying-Squirrel & & & & 1 & & & & & & - & \\
\hline American Beaver & & & & & & & 인 & & & o & \\
\hline Deer Mouse & & & $\mathrm{t}$ & & & & $\mathrm{t}$ & & & & \\
\hline Muskrat & & & 1 & & & & & & & & \\
\hline \multicolumn{12}{|l|}{ Gapper's Red-Backed Vole } \\
\hline Meadow Vole & & & & & & & & & & $t$ & \\
\hline \multicolumn{12}{|l|}{ Vole Species } \\
\hline \multicolumn{12}{|l|}{ Mouse Species } \\
\hline \multicolumn{12}{|l|}{ Norway Rat } \\
\hline \multicolumn{12}{|l|}{ House Mouse } \\
\hline American Porcupine & & & & 1 & & & & & & & $\mathrm{t}$ \\
\hline Coyote & $\mathrm{t}$ & & $\mathrm{t}$ & $t$ & 15 & & 2 & & 1 & 5 & 1 \\
\hline Wolf & $\mathrm{t}$ & & & $\mathrm{t}$ & $\mathrm{t}$ & & & & & & \\
\hline Red Fox & & 2 & $\mathrm{t}$ & 1 & & & $\mathrm{t}$ & & & $t$ & 0 \\
\hline \multicolumn{12}{|l|}{ Swift Fox } \\
\hline \multicolumn{12}{|l|}{ Raccoon } \\
\hline \multicolumn{12}{|l|}{ American Marten } \\
\hline \multicolumn{12}{|l|}{ Fisher } \\
\hline \multicolumn{12}{|l|}{ Ermine } \\
\hline \multicolumn{12}{|l|}{ Long-tailed Weasel } \\
\hline \multicolumn{12}{|l|}{ Least Weasel } \\
\hline Weasel Species & & & t) & & 1 & & 1 & & & & \\
\hline \multicolumn{12}{|l|}{ American Mink } \\
\hline American Badger & & & & & & & 아 & & & & 0 \\
\hline \multicolumn{12}{|l|}{ Striped Skunk } \\
\hline \multicolumn{12}{|l|}{ River Otter } \\
\hline Lynx & & & & & & & & & & & \\
\hline Woodland Caribou & & & & & & & & & & & \\
\hline Mule Deer & & & 25 & $\mathrm{t}$ & & & c & & 10 & & \\
\hline White-tailed Deer & 5 & 8 & 18 & 6 & 14 & 15 & 1 & 29 & & 8 & $\mathrm{t}$ \\
\hline Deer Species & & & & & & & & & & & \\
\hline Moose & & & & & $\mathrm{t}$ & & 3 & & & $t$ & \\
\hline Elk & 75 & & & $\mathrm{t}$ & & & & & & & \\
\hline Pronghorn & & & & & & & & & & & \\
\hline Totals seen/heard on count day & 80 & 16 & 71 & 15 & 41 & 21 & 13 & 35 & 12 & 15 & 6 \\
\hline Total species seen/heard & 2 & 3 & 7 & 5 & 4 & 4 & 5 & 3 & 3 & 3 & 4 \\
\hline Total species recorded by tracks & 6 & 0 & 5 & 5 & 2 & 0 & 5 & 0 & 0 & 6 & 2 \\
\hline Total species otherwise recorded & 0 & 0 & 1 & 0 & 0 & 0 & 2 & 0 & 0 & 1 & 2 \\
\hline Species recorded count period & 0 & 0 & 0 & 0 & 0 & 0 & 1 & 0 & 0 & 2 & 0 \\
\hline Total species count period and day & 8 & 3 & 13 & 10 & 6 & 4 & 13 & 3 & 3 & 12 & 8 \\
\hline
\end{tabular}


Table 1. 32 $2^{\text {nd }}$ Saskatchewan Christmas Mammal Count-2004.

\begin{tabular}{|c|c|c|c|c|c|c|}
\hline & 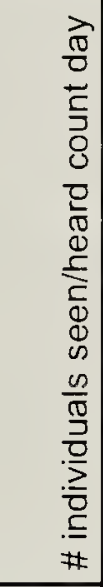 & $\begin{array}{l}\text { D } \\
0 \\
\Phi \\
5 \\
0 \\
0 \\
0 \\
0 \\
0 \\
5 \\
0 \\
0 \\
\# \\
\end{array}$ & 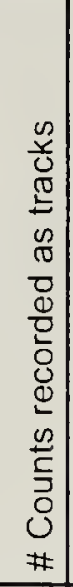 & $\begin{array}{l}\overline{0} \\
\bar{t} \\
0 \\
i \\
0 \\
0 \\
0 \\
0 \\
0 \\
0 \\
0 \\
0 \\
0 \\
5 \\
0 \\
0 \\
\#\end{array}$ & 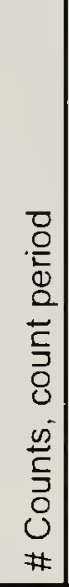 & 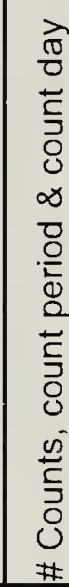 \\
\hline Short-tailed Shrew & 1 & 1 & 1 & 0 & 0 & 2 \\
\hline Shrew Species & 0 & 0 & 2 & 0 & 0 & 2 \\
\hline Nuttall's Cottontail & 48 & 13 & 5 & 0 & 0 & 18 \\
\hline Snowshoe Hare & 73 & 16 & 25 & 0 & 1 & 42 \\
\hline White-tailed Jack Rabbit & 124 & 27 & 13 & 0 & 1 & 41 \\
\hline Least Chipmunk & 1 & 1 & 0 & 0 & 0 & 1 \\
\hline Eastern Grey Squirrel & 24 & 5 & 0 & 0 & 1 & 6 \\
\hline Fox Squirrel & 18 & 6 & 0 & 0 & 1 & 7 \\
\hline American Red Squirrel & 275 & 50 & 5 & 0 & 1 & 56 \\
\hline Northern Fiying-Squirrel & 1 & 1 & 0 & 0 & 1 & 2 \\
\hline American Beaver & 10 & 2 & 0 & 13 & 1 & 16 \\
\hline Deer Mouse & 6 & 4 & 9 & 0 & 2 & 15 \\
\hline Muskrat & 4 & 4 & 0 & 3 & 0 & 7 \\
\hline Gapper's Red-Backed Vole & 0 & 0 & 1 & 0 & 0 & 1 \\
\hline Meadow Vole & 2 & 2 & 10 & 1 & 1 & 14 \\
\hline Vole Species & 9 & 7 & 4 & 0 & 0 & 11 \\
\hline Mouse Species & 6 & 1 & 11 & 0 & 0 & 12 \\
\hline Norway Rat & -0 & 0 & 2 & 0 & 0 & 2 \\
\hline House Mouse & 2 & 2 & 0 & 0 & 1 & 3 \\
\hline American Porcupine & 14 & 8 & 12 & 4 & 1 & 25 \\
\hline Coyote & 178 & 56 & 17 & 0 & 2 & 75 \\
\hline Wolf & 2 & 1 & 6 & 0 & 0 & 7 \\
\hline Red Fox & 33 & 19 & 14 & 2 & 7 & 42 \\
\hline Swift Fox & 0 & 0 & 0 & 1 & 0 & 1 \\
\hline Raccoon & 3 & 2 & 4 & 0 & 0 & 6 \\
\hline American Marten & 2 & 1 & 2 & 0 & 0 & 3 \\
\hline Fisher & 1 & 1. & 2 & 0 & 0 & 3 \\
\hline Ermine & 3 & 3 & 6 & 0 & 0 & 9 \\
\hline Long-tailed Weasel & 10 & 5 & 7 & 0 & 0 & 12 \\
\hline Least Weasel & 1 & 1 & 4 & 0 & 0 & 5 \\
\hline Weasel Species & 4 & 3 & 10 & 0 & 2 & 15 \\
\hline American Mink & 0 & 0 & 3 & 0 & 1 & 4 \\
\hline American Badger & 2 & 2 & 0 & 7 & 0 & 9 \\
\hline Striped Skunk & 2 & 1 & 1 & 4 & 1 & 7 \\
\hline River Otter & 2 & 1 & 1 & 0 & 0 & 2 \\
\hline Lynx & 2 & 2 & 2 & 0 & 0 & 4 \\
\hline Woodland Caribou & 0 & 0 & 1 & 0 & 0 & 1 \\
\hline Mule Deer & 1062 & 37 & 3 & 0 & 3 & 43 \\
\hline White-tailed Deer & 1403 & 67 & 13 & 0 & 1 & 81 \\
\hline Deer Species & 50 & 3 & 1 & 0 & 0 & 4 \\
\hline Moose & 18 & 8 & 9 & 0 & 3 & 20 \\
\hline Elk & 181 & 5 & 7 & 0 & 1 & 13 \\
\hline Pronghorn & 217 & 6 & 1 & 0 & 0 & 7 \\
\hline Totals seen/heard on count day & 3794 & & & & & \\
\hline Total species seen/heard & & 33 & & & & \\
\hline Total species recorded by tracks & & & 30 & & & \\
\hline Total species otherwise recorded & & & & 8 & & \\
\hline Species recorded count period & & & & & 19 & \\
\hline Total species count period and day & & & & & & 39 \\
\hline
\end{tabular}

